\title{
Complexity of factors related to outcome of neuropathic and neuroischaemic/ischaemic diabetic foot ulcers: a cohort study
}

\author{
M. A. Gershater • M. Löndahl • P. Nyberg • J. Larsson • \\ J. Thörne • M. Eneroth • J. Apelqvist
}

Received: 24 July 2008 / Accepted: 28 October 2008 / Published online: 27 November 2008

(C) Springer-Verlag 2008

\begin{abstract}
Aims/hypothesis We sought to identify factors related to shortterm outcome of foot ulcers in patients with diabetes treated in a multidisciplinary system until healing was achieved.

Methods Consecutively presenting patients with diabetes and worst foot ulcer (Wagner grade 1-5, below ankle) $(n=2,511)$ were prospectively followed and treated according to a standardised protocol until healing was achieved or until death. The number of patients lost to dropout was 31 .
\end{abstract}

M. A. Gershater $(\bowtie)$

Faculty of Health and Society, Malmö University, 20506 Malmö, Sweden

e-mail: magdalena.gershater@mah.se

M. Löndahl

Department of Endocrinology/Diabetology,

Lund University Hospital,

Lund, Sweden

P. Nyberg

Faculty of Medicine, Department of Health Sciences,

Lund University,

Lund, Sweden

J. Larsson

Department of Orthopaedic Surgery, Lund University Hospital,

Lund, Sweden

J. Thörne

Department of Surgery, Helsingborg Hospital,

Helsingborg, Sweden

M. Eneroth

Department of Orthopaedic Surgery, Malmö University Hospital, Malmö, Sweden

J. Apelqvist

Department of Endocrinology, Malmö University Hospital,

Malmö, Sweden
The characteristics of the remaining 2,480 patients were: 1,465 men, age $68 \pm 15$ years (range 18-96), type 1 diabetes $18 \%$, type 2 diabetes $82 \%$ and insulin-treated $62 \%$.

Results The healing rate without major amputation in surviving patients was 90.6\% $(n=1,867)$. Sixty-five per cent $(n=1,617)$ were healed primarily, 9\% $(n=250)$ after minor amputation and $8 \%$ after major amputation; $17 \%$ $(n=420)$ died unhealed. Out of 2,060 surviving patients, 1,007 were neuroischaemic $(48.8 \%)$. In a multiple regression analysis, primary healing was related to co-morbidity, duration of diabetes, extent of peripheral vascular disease and type of ulcer. In neuropathic ulcers, deep foot infection, site of ulcer and co-morbidity were related to amputation. Amputation in neuroischaemic ulcers was related to comorbidity, peripheral vascular disease and type of ulcer. Age, sex, duration of diabetes, neuropathy, deformity and duration of ulcer or site of ulcer did not have an evident influence on probability of amputation.

Conclusions/interpretation Patients with diabetic foot ulcer suffer from multi-organ disease. Factors related to outcome are correspondingly complex.

Keywords Amputation - Complications · Diabetes mellitus · Diabetic foot ulcers $\cdot$ Healing $\cdot$ Neuropathy $\cdot$ Peripheral vascular disease

\section{Abbreviation \\ PVD peripheral vascular disease}

\section{Introduction}

Foot complications in diabetes present a particularly troubling picture. Indeed, it has been claimed that every 
$30 \mathrm{~s}$ a lower limb is amputated somewhere in the world due to diabetes [1]. The diabetic foot can be defined as ulceration associated with neurological abnormalities, infection and various degrees of peripheral vascular disease (PVD) [2]. Some $85 \%$ of diabetes-related amputations are preceded by a foot ulcer $[2,3]$. The number of large cohort studies presenting data on outcomes and determinants of outcome in individuals with diabetic foot ulcers is limited [1, 2, 4-6].

The present study was designed to obtain prospective data on outcome and determinants of outcome of foot ulcers, focusing on individual and disease-specific factors in patients with diabetes treated in a multidisciplinary system.

\section{Methods}

\section{Study population}

Patients consecutively presenting with diabetes and a foot ulcer at a multidisciplinary foot centre $(n=2,831)$ were included in this cohort study and prospectively followed, treated and recorded according to a standardised protocol (see below) until healing was achieved or until death, irrespective of time.

\section{Inclusion criteria}

Inclusion criteria included: age 18 years or older, diabetes mellitus and foot ulcer (a full-thickness ulcer penetrating the subcutaneous layer, Wagner grade 1-5) located at or below the ankle [7]. Patients were excluded due to: (1) violation of inclusion criteria $(n=315)$, i.e. diabetes not confirmed, ulcer above ankle or lesions involving skin only; and (2) dropout $(n=31)$, e.g. patients moved to other areas or were lost to home nursing service. The remaining 2,480 patients were followed to outcome according to protocol. Patient consent was given at inclusion into the study.

A pre-set standardised protocol was designed prior to the study and was used at inclusion and throughout the study. All lesions were assessed and documented by the same team.

\section{Definitions}

Ulcer Each patient was represented by one lesion below the ankle. In all cases, the most severe ulcer occurring during the observation period was described. Patients with several concurrent lesions were represented by that with the worst outcome. Patients with three or more ulcers on the same foot were defined as having 'multiple ulcers'. Lesions were classified according to Wagner [7, 8].

Wound healing This was defined as intact skin for 6 months or at time of death.
Outcome Several outcomes were defined: (1) primary healed, defined as healed without any amputation with intact skin for 6 months or intact skin at time of death; (2) minor amputation, defined as amputation of one or more toes or some part of the foot at or below the ankle; (3) major amputation, defined as amputation above the ankle; and (4) deceased unhealed, defined as death without healing with or without any amputation.

Diabetes mellitus This was defined arbitrarily as type 1 diabetes if diagnosed before 30 years of age and as type 2 diabetes if age at diagnosis was 30 years or more.

Vascular disease, circulatory disorders Hypertension, angina pectoris, myocardial infarction, congestive heart failure, non-ischaemic heart disease and cerebrovascular disease were defined as previously described [9].

Retinopathy and nephropathy The former was defined as previously described [9] after fundus photography by an ophthalmologist. Non-retinal eye diseases were also recorded. Nephropathy was considered present at persistent urine albumin $>300 \mathrm{mg} / 1$ [9].

Foot disorders Deformity was defined as claw toes with hyperextension in the metatarso-phalangeal joint and flexion of the distal inter-phalangeal joint, hallux valgus and hallux rigidus. Sensory/motoric neuropathy was defined as vibratory pressure threshold values $>30$ with concomitant muscle-wasting. Oedema was considered present when swelling of the foot was so pronounced as to leave imprint after pressure by a finger. Deep foot infection was defined as one of the following conditions: deep abscess (Wagner grade 3 [7]) or deep soft tissue infection with or without osteomyelitis. Osteomyelitis was diagnosed in all patients with an open lesion fulfilling at least three of the following criteria: cellulitis, positive bacterial culture, radiological or scintigraphic evidence and histological diagnosis [10]. Gangrene was defined as a continuous necrosis of the skin and underlying structures indicating irreversible damage that would be unlikely to heal without loss of some part of the extremity (Wagner grades 4-5) [7].

Rest pain This was defined as severe persistent pain localised to the foot and relieved by lowering of the foot.

Claudication Recurrent cramping pain or tightness in the calf induced by exercise and relieved by rest [11, 12] was defined as claudication.

Neuroischaemic/ischaemic ulcers These were considered present at ankle pressure $<80 \mathrm{mmHg}$ or toe pressure 
$<45 \mathrm{mmHg}$ or at Wagner grades 4 to 5 if distal pressure was not obtained [13].

Duration of ulcer Duration was defined as the estimated number of weeks from development of ulcer until entry in the study.

Other factors Assisted living was defined as any institutional living outside the patient's own home. Smoking habits were classified according to whether the patient had ever smoked regularly or not. In the former case, he/she was considered a smoker. Compliance was defined as the ability to follow given prescriptions regarding medical treatment, to use off-loading equipment and to attend the multidisciplinary team visits.

\section{Organisation and setting}

The patients were consecutively recruited, followed and treated by the multidisciplinary team at Malmö and Lund University Hospitals both as in- and outpatients until healing was achieved. Outpatient treatment was carried out in collaboration with primary healthcare and home nursing services. Physical examination was performed at inclusion and regularly during the study by the multidisciplinary team. The core team consisted of a diabetologist, orthopaedic surgeon, orthotist, podiatrist and a registered nurse educated in diabetes. Vascular investigation was carried out according to a prescheduled programme by a vascular surgeon integrated in the team on a regular basis. Specially trained casting technicians provided continuous service for total contact casting. A specialist in infectious disease was available for consultations when required. A registered nurse coordinated the team, performed care planning and patient education.

\section{Medical treatment}

Patients were offered medical treatment to improve metabolic control and optimise treatment of co-morbidity [2]. Patients needing such treatment were also given nutrition and hydration treatment [14]. When clinical signs of infection were present, oral treatment with antibiotics was provided, often combining cephalosporin, quinolone or dicloxacillin with metronidazole or clindamycin. In patients with deep abscess or acute osteomyelitis, the patient was hospitalised and intravenous antibiotics used [15]. A differentiated programme for analgesia was used related to cause and intensity of pain.

\section{Surgical treatment}

Surgery was performed when deemed necessary by an orthopaedic surgeon. Minor debridements were performed in the outpatient clinic; for major revisions and amputations the patient was admitted to the hospital [16]. A non-healing ulcer was not an indication for amputation, the criteria being progressive gangrene, intolerable pain despite adequate analgesic medication, and septic and toxic conditions not responding to medical treatment [3]. Vascular intervention was performed at the discretion of a vascular surgeon according to a written programme that was jointly agreed upon.

\section{Off-loading}

All patients were offered off-loading equipment adjusted to their individual needs. Total contact casts, orthoses, insoles, specially made shoes, half shoes or wheel chairs were used. Off-loading by crutches was occasionally used.

\section{Topical treatment}

According to the individual wound bed condition, different topical treatment was prescribed in written form by the multi-disciplinary team. Dressing changes were performed under supervision of a registered nurse in primary healthcare or home nursing services. The team maintained daytime telephone service for support. Most commonly used dressings were: foam dressings, hydro-fibre, hydrogels, matrix stimulating factors (hyaluronic acid), siliconenet or hydrophobic gauze. Silver and cadexomere iodine were used as topical antimicrobial agents when appropriate. External compression bandages or intermittent compression therapies were used in the presence of peripherally induced oedema [2].

\section{Measurements}

Ulcer size was estimated in millimetres using a ruler and dichotomised into $<1 \mathrm{~cm}^{2}$ or larger. Volume was not measured. Systolic toe and ankle blood pressure was measured using strain gauge and Doppler techniques at the vascular laboratory [13]. Signs of sensory polyneuropathy were tested using biothesiometer (BioMedical Instruments, New Burry, OH, USA) and defined as present at biothesiometer values of $30 \mathrm{~V}$ or more.

\section{Data management}

At study entry data were collected on previous disease management, referral, patient characteristics, co-morbidities and ulcer characteristics. Data were recorded continuously during follow-up visits to the multidisciplinary team using standardised case record forms; these forms were computerised into an Access database and transformed into files. 
The database was closed for analysis on 31 December 2005.

Statistics

A multiple regression analysis was performed including factors of demographic data, clinical characteristics, comorbidity and local characteristics as well as social factors $(n=2,480)$. Statistical significance was defined as a $p$ value $<0.05$. Retinopathy was excluded from the model. Patients $(n=142)$ with one or more factor not available were excluded. Values are given as median and range analysed with the Mann-Whitney test [17]. The simultaneous influence of possible risk factors on a binary outcome (primary healing and amputation) was investigated by means of backward logistic regression analysis using the Hosmer and Lemeshow test for goodness of fit. Statistical analysis was performed using SPSS version 14.0 (SPSS, Chicago, IL, USA).

\section{Ethical approval}

The Regional Ethical Review Board in Lund approved the study.

\section{Results}

Patient characteristics and overall outcome

As shown in Table 1, the healing rate without major amputation in surviving patients was $90.6 \%(n=1,867)$.

Primary healing was seen in two thirds of all ulcers: $65 \%$ $(n=1,617)$; $9 \%(n=250)$ healed after minor amputation; $8 \%$ healed after major amputation $(n=193$, transfemoral $n=19)$; and $17 \%$ deceased unhealed $(n=420$; including 43 amputated prior to death). Time to healing was median 18 weeks (1-235). Neuropathic ulcers constituted 59\% $(n=1,473)$ and neuroischaemic/ischaemic $41 \%(n=1,007)$ of all ulcers $(p<0.001)$.

Duration of diabetes did not differ between the four groups. In patients who died unhealed, there was a higher proportion of type 2 diabetes (93\%), but the distribution between type 1 and type 2 diabetes did not differ between the surviving groups. The proportion of men and women did not differ between the groups. Due to their medical condition, 442 patients had no eye examination. Heart disease (ischaemic heart disease, congestive heart failure or other cardiac disease) affected $53 \%$ of the patients. Patients who underwent major amputation or died before healing

Table 1 General patient characteristics at enrolment

\begin{tabular}{|c|c|c|c|c|c|}
\hline Characteristic & Primary healed & Minor amputation & Major amputation & Deceased unhealed & All \\
\hline$n$ & 1,617 & 250 & 193 & 420 & 2,480 \\
\hline Men & $945(58)$ & $171(68)$ & $106(55)$ & $243(58)$ & $1,465(59)$ \\
\hline Women & $672(42)$ & $79(31)$ & $87(45)$ & $177(42)$ & $1,015(41)$ \\
\hline Age (years) ${ }^{\mathrm{a}}$ & $66(18-96)$ & $68(26-95)$ & $72(39-93)$ & $77(34-96)$ & $68(18-96)$ \\
\hline$p$ value & & 0.502 & $<0.001$ & $<0.001$ & \\
\hline Type 1 diabetes & $350(22)$ & $44(18)$ & $28(15)$ & $30(7)$ & $(18)$ \\
\hline Type 2 diabetes & $1,267(78)$ & $206(72)$ & $165(85)$ & $390(93)$ & $(82)$ \\
\hline Insulin-treated & $1,001(62)$ & $161(64)$ & $123(64)$ & $243(58)$ & $(61.5)$ \\
\hline Diabetes duration (years) ${ }^{\mathrm{a}}$ & $16(0-62)$ & $17(0-70)$ & $17(0-58)$ & $16(0-58)$ & $16(0-70)$ \\
\hline$p$ value & & 0.165 & 0.120 & 0.762 & \\
\hline Proliferative retinopathy & $639(40)$ & $116(46)$ & $83(43)$ & $190(45)$ & $(41)^{\mathrm{b}}$ \\
\hline Nephropathy & $411(25)$ & $91(36)$ & $57(30)$ & $126(30)$ & $(28)$ \\
\hline Uraemia & $89(5)$ & $23(9)$ & $28(15)$ & $67(16)$ & (8) \\
\hline Hypertension & $882(55)$ & $140(56)$ & $110(57)$ & $249(59)$ & $(56)$ \\
\hline Ischaemic heart disease & $400(25)$ & $75(30)$ & $78(40)$ & $206(49)$ & $(30)$ \\
\hline Congestive heart failure & $272(16)$ & 48 (19) & $64(33)$ & $190(45)$ & (23) \\
\hline Cerebrovascular disease & $310(19)$ & 48 (19) & $53(27)$ & $163(39)$ & (23) \\
\hline Never-smokers & $657(41)$ & $79(32)$ & $81(42)$ & $185(44)$ & $(40)$ \\
\hline Assisted living & $197(12)$ & $25(10)$ & $39(20)$ & $161(38)$ & (17) \\
\hline Non-compliant & $191(12)$ & 47 (19) & $17(9)$ & $31(7)$ & $(10)$ \\
\hline Neuropathic & $1,170(79.4)$ & $104(7.1)$ & $36(2.4)$ & $163(11.1)$ & $1,473(100)$ \\
\hline Neuroischaemic/ischaemic & $447(44.4)$ & $146(14.5)$ & $157(15.6)$ & $257(25.5)$ & $1,007(100)$ \\
\hline
\end{tabular}

Values are $n(\%)$ (per cent values were calculated on all patients per status group)

${ }^{a}$ Mean value (range)

${ }^{\mathrm{b}}$ Data missing for 18 patients 
Table 2 Local characteristics of the foot at enrolment

\begin{tabular}{|c|c|c|c|c|c|}
\hline Characteristic & $\begin{array}{l}\text { Primary } \\
\text { healed }\end{array}$ & $\begin{array}{l}\text { Minor } \\
\text { amputation }\end{array}$ & $\begin{array}{l}\text { Major } \\
\text { amputation }\end{array}$ & $\begin{array}{l}\text { Deceased } \\
\text { unhealed }\end{array}$ & All \\
\hline Peripheral neuropathy & $1,482(92)$ & $230(92)$ & $177(92)$ & $388(92)$ & $(92)$ \\
\hline Foot deformity & $1,238(77)$ & $230(79)$ & $149(77)$ & $323(77)$ & (77) \\
\hline Big toe & $450(28)$ & $61(24)$ & $29(15)$ & $94(22)$ & $634(26)$ \\
\hline Other toe & $273(14)$ & $76(30)$ & $37(19)$ & $45(11)$ & $431(17)$ \\
\hline Plantar ulcer & $253(16)$ & $48(19)$ & $10(5)$ & $46(11)$ & $(13)$ \\
\hline Multiple ulcer & $102(6)$ & $36(14)$ & $68(35)$ & $100(24)$ & (17) \\
\hline Heel ulcer & $276(17)$ & $4(2)$ & $28(15)$ & $76(18)$ & $384(15)$ \\
\hline Other location & $263(16)$ & $25(10)$ & $21(11)$ & $59(14)$ & $368(15)$ \\
\hline Ulcer size $<1 \mathrm{~cm}^{2}$ & $370(23)$ & $48(19)$ & $22(11)$ & $55(13)$ & $495(20)$ \\
\hline Traumatic ulcer/stress ulcer & $942(58)$ & $149(60)$ & $66(34)$ & $150(36)$ & $1,307(53)$ \\
\hline Fissure & $88(5)$ & $8(3)$ & $6(3)$ & $14(3)$ & $116(5)$ \\
\hline Paronychia & $86(5)$ & $5(2)$ & $5(3)$ & $17(4)$ & $113(5)$ \\
\hline Reason unknown & $415(26)$ & $85(34)$ & $101(52)$ & $178(42)$ & $779(31)$ \\
\hline Pressure ulcer & $86(5)$ & $3(1)$ & $15(8)$ & $61(14)$ & $165(7)$ \\
\hline Wagner grade 3 & $115(7)$ & $81(41)$ & $23(9)$ & $27(6)$ & (10) \\
\hline Wagner grade 4-5 & $29(2)$ & $37(15)$ & $46(23)$ & $44(10)$ & (6) \\
\hline \multicolumn{6}{|l|}{ Ankle pressure $(\mathrm{mmHg})$} \\
\hline$<80$ & $264(16)$ & $66(26)$ & $75(39)$ & $156(37)$ & $(22)$ \\
\hline$<50$ & $88(5)$ & $34(14)$ & $38(29)$ & $64(15)$ & (16) \\
\hline \multicolumn{6}{|l|}{ Toe pressure $(\mathrm{mmHg})$} \\
\hline$<45$ & $327(20)$ & $90(36)$ & $106(55)$ & $187(45)$ & $(29)$ \\
\hline$<30$ & $161(10)$ & $56(22)$ & $77(40)$ & $130(31)$ & (17) \\
\hline Previous amputation & $57(3.5)$ & $18(7)$ & $27(14)$ & $43(10)$ & (6) \\
\hline Previous vascular surgery & $72(4.4)$ & $19(7.6)$ & $21(11)$ & $34(8)$ & (8) \\
\hline Oedema & $536(33)$ & $161(64)$ & $121(63)$ & $212(50)$ & (41) \\
\hline Rest pain & $294(18)$ & $92(37)$ & $114(59)$ & $164(39)$ & $(27)$ \\
\hline Duration of ulcer at inclusion (weeks) ${ }^{\mathrm{a}}$ & $10(0-260)$ & $10(1-156)$ & $14(0-350)$ & $13(0-208)$ & $11(0-350)$ \\
\hline Duration of ulcer from inclusion to healing (weeks) ${ }^{\mathrm{a}}$ & $15.0(1-204)$ & $34.9(1-235)$ & $28.0(1-170)$ & & $(1-235)$ \\
\hline
\end{tabular}

Values are $n(\%)$

${ }^{\mathrm{a}}$ Values are median (range)

had significantly more cardiovascular complications and more lived in assisted living (thus being dependent on others to cope with their daily life) than patients who healed without amputation or with minor amputation. In patients older than 80 years of age $(n=630)$, the primary healing rate was $51 \%(n=323)$, amputation rate $16 \%$ (minor amputation $n=45$, major amputation $n=54$ ) and those deceased unhealed 33\% $(n=206)$.

\section{Ulcer characteristics}

As seen in Table 2, the estimated duration of ulcer before presentation to the multidisciplinary team was $<4$ weeks in $46 \%, 4$ to 26 weeks in $43 \%$ and more than 26 weeks in $11 \%$ of patients. In $70 \%$ of patients presenting to the team, external, precipitating factors could be established, the most common being acute trauma (20\%), ill-fitting shoes (19\%) and stress ulcer $(18 \%)$. The big toe $(n=634,26 \%)$ was the most common site of ulcer and $13 \%(n=315)$ of ulcers were located to the metatarsal heads. Out of 2,060 surviving patients, 1,007 had neuroischaemic/ischaemic ulcers (48.8\%). Out of 561 patients with ankle pressure $<80 \mathrm{mmHg}$, 264 healed during primary treatment. The corresponding number for the 224 patients with ankle pressure below $50 \mathrm{mmHg}$ was 88 and those for the 710 patients with a toe pressure $<45 \mathrm{mmHg}$ or the 307 patients $<30 \mathrm{mmHg}$ were 327 and 161 respectively. Of 664 patients with rest pain, 44\% healed without any amputation.

Factors related to primary healing

In the regression analysis, primary healed patients $(n=1,617)$ were compared with the remaining cohort and surviving patients with minor and major amputations $(n=443)$ (Tables 3 and 4). By means of backward logistic regression, removing non-significant variables in 17 steps, the remaining variables are presented in Tables 3, 4, 5, 6 . Primary healing was related to duration of diabetes, comorbidity, good compliance and single ulcer (Tables 3, 4, 5 and 6). Further regression analysis was performed 
Table 3 Factors related to primary healing

\begin{tabular}{lrrr}
\hline Factor & Odds ratio & $95 \%$ CI & $p$ value \\
\hline Duration of diabetes & & & \\
0-7 years & 1.71 & $1.02-2.87$ & 0.041 \\
8-15 years & 2.12 & $1.27-3.56$ & 0.004 \\
Good compliance & 1.62 & $1.04-2.52$ & 0.034 \\
Absence of non-retinal eye & 1.60 & $1.11-2.30$ & 0.012 \\
$\quad$ disease & & & \\
Absence of uraemia & 2.43 & $1.35-4.38$ & 0.003 \\
Absence of any heart disease & 1.44 & $1.03-2.01$ & 0.032 \\
No previous amputation & 3.70 & $1.91-7.18$ & 0.000 \\
No intermittent claudication & 1.84 & $1.14-2.97$ & 0.012 \\
No rest pain & 1.59 & $1.11-2.27$ & 0.011 \\
Toe pressure $>45$ mmHg & 1.42 & $1.00-2.04$ & 0.050 \\
No oedema & 1.63 & $1.17-2.27$ & 0.004 \\
Single ulcer & 2.10 & $1.28-3.45$ & 0.003 \\
Wagner grade 1-2 & 39.08 & $25.63-59.57$ & 0.000 \\
Wagner grade 1-3 & 4.32 & $2.79-6.70$ & 0.000 \\
\hline
\end{tabular}

regarding probability in surviving patients with neuropathic or neuroischaemic/ischaemic ulcers (Tables 5 and 6). There were corresponding factors related to healing in these groups.

Factors related to amputation in neuropathic ulcers

Patients with neuropathic ulcers $(n=661)$ were included in the regression analysis where healing after minor or major amputation in neuropathic ulcers was related to comorbidity, type 2 diabetes, duration of diabetes 8 to 23 years, visual impairment, uraemia, oedema, previous amputation (of toe or leg), walking disability, rest pain, plantar ulcer and deep infection (Table 7).

Table 4 Factors related to primary healing in all surviving patients (model with all surviving patients)

\begin{tabular}{lccr}
\hline Factor & Odds ratio & \multicolumn{1}{l}{$95 \% \mathrm{CI}$} & $p$ value \\
\hline Duration of diabetes & & & \\
0-7 years & 1.68 & $1.09-2.28$ & 0.017 \\
8-15 years & 1.80 & $1.17-2.77$ & 0.007 \\
No uraemia & 1.94 & $1.14-3.29$ & 0.015 \\
No previous amputation & 3.75 & $2.08-6.77$ & $<0.005$ \\
No heart disease & 1.39 & $1.02-1.89$ & 0.023 \\
Toe pressure $>$ 45 mmHg & 1.42 & $1.03-1.96$ & 0.024 \\
No oedema & 1.56 & $1.14-2.12$ & 0.005 \\
No pain & 1.81 & $1.30-2.51$ & $<0.005$ \\
Single ulcer & 2.06 & $1.32-3.27$ & 0.002 \\
Wagner grade 1 and 2 & 36.30 & $23.90-52.80$ & $<0.005$ \\
Wagner grade 1, 2 and 3 & 4.12 & $2.57-5.97$ & $<0.05$ \\
\hline
\end{tabular}

Table 5 Factors related to primary healing in all surviving neuropathic patients

\begin{tabular}{lrrr}
\hline Factor & $\begin{array}{l}\text { Odds } \\
\text { ratio }\end{array}$ & $95 \%$ CI & $p$ value \\
& 2.77 & $1.67-4.60$ & $<0.005$ \\
\hline Age 18-60 years & 1.93 & $1.22-3.04$ & 0.005 \\
Age 71-80 years & 1.72 & $1.10-2.67$ & 0.017 \\
Duration of diabetes 0-7 years & 1.80 & $1.16-2.79$ & 0.009 \\
Duration of diabetes & & & \\
$\quad$ 8-15 years & 2.16 & $1.43-3.28$ & $<0.005$ \\
Living in own home & 2.61 & $1.57-4.35$ & $<0.005$ \\
No uraemia & 2.89 & $1.49-5.61$ & 0.002 \\
No previous amputation & 2.11 & $1.54-2.90$ & $<0.005$ \\
Absence of any heart disease & 1.57 & $1.15-2.14$ & 0.005 \\
No oedema & 1.85 & $1.08-3.17$ & 0.025 \\
Vascular surgery & 1.89 & $1.12-3.17$ & 0.017 \\
Single ulcer & 16.55 & $9.99-27.42$ & $<0.005$ \\
Wagner grade 1 and 2 & 3.91 & $2.24-6.82$ & $<0.005$ \\
Wagner grade 1, 2 and 3 & & & \\
\hline
\end{tabular}

Factors related to major amputation in neuroischaemic/ischaemic ulcers

Patients with neuroischaemic/ischaemic ulcers (49\%, $n=1,007)$ were included in the regression analysis where factors related to major amputation were identified: duration of diabetes $>23$ years, uraemia, oedema, foot deformity, peripheral arterial disease, multiple ulcers, male sex and noncompliance (Table 8). Wagner grades 4 to 5 were excluded from the analysis due to self-evident definition.

\section{Discussion}

This study with a healing rate of $90.6 \%$ without major amputation in surviving patients is the largest prospective

Table 6 Factors related to primary healing in all surviving neuroischaemic/ischaemic patients

\begin{tabular}{lccr}
\hline Factor & Odds ratio & $95 \%$ CI & $p$ value \\
\hline Age 18-60 years & 1.80 & $1.01-3.21$ & 0.045 \\
Age 71-80 years & 1.70 & $1.14-2.52$ & 0.009 \\
Male sex & 1.40 & $1.00-1.93$ & 0.044 \\
Living in own home & 2.43 & $1.58-3.72$ & $<0.005$ \\
Nephropathy & 1.54 & $1.03-2.28$ & 0.033 \\
No uraemia & 2.45 & $1.35-4.46$ & 0.003 \\
No previous amputation & 2.30 & $1.23-4.29$ & 0.009 \\
Claudication & 1.71 & $1.17-2.51$ & 0.006 \\
No pain & 1.90 & $1.37-2.63$ & $<0.005$ \\
Single ulcer & 2.17 & $1.44-3.28$ & $<0.005$ \\
Wagner grade 1 and 2 & 9.76 & $6.77-14.08$ & $<0.005$ \\
Wagner grade 1, 2 and 3 & 3.52 & $2.11-5.88$ & $<0.005$ \\
\hline
\end{tabular}


Table 7 Factors related to minor or major amputation in neuropathic ulcers

\begin{tabular}{lccc}
\hline Factor & $\begin{array}{c}\text { Odds } \\
\text { ratio }\end{array}$ & $95 \%$ CI & $p$ value \\
& 1.94 & $1.08-3.49$ & 0.026 \\
Type 2 diabetes & 1.91 & $1.11-3.28$ & 0.020 \\
Duration of diabetes 8-15 years & 2.66 & $1.43-4.95$ & 0.002 \\
Duration of diabetes 15-23 years & 1.85 & $1.16-2.85$ & 0.009 \\
Non-retinal eye disease/visual & & & \\
$\quad$ impairment & 2.62 & $1.39-4.96$ & 0.003 \\
Uraemia & 2.07 & $1.40-3.05$ & 0.000 \\
Oedema & 3.33 & $1.54-7.22$ & 0.002 \\
Previous amputation & 1.71 & $1.16-2.52$ & 0.007 \\
Walking disability & 2.52 & $1.66-3.83$ & 0.000 \\
Rest pain & 4.13 & $2.34-7.28$ & 0.000 \\
Plantar ulcer & 4.80 & $3.13-7.34$ & 0.000 \\
Deep infection $^{\mathrm{a}}$ & & & \\
\hline
\end{tabular}

${ }^{\text {a }}$ Wagner grade 3 , osteomyelitis, abscess

cohort study of diabetic patients who had a foot ulcer treated by a multi-disciplinary foot centre and who were consecutively recruited and followed until healing or death.

The study identified a complexity of factors related to outcome, of which co-morbidity, duration of disease, extent of tissue involvement and extent of PVD were strongly related to probability of primary healing. These findings underline the need for recognition of diabetic foot ulcers as a sign of underlying multi-organ disease.

The number of large cohort studies with factors related to healing of foot ulcers is limited. In mixed cohort studies primary healing rates of 60 to $74 \%$ and amputation rates of 8 to $23 \%$ have been reported $[4,18,19]$. In the present study, the primary healing rate in the whole cohort without any amputation was $65 \%$ and the mortality rate of unhealed patients was $16.9 \%$. These findings are in agreement with some, but not all previous studies, with Prompers et al.

Table 8 Factors related to major amputation in neuroischaemic/ ischaemic ulcers

\begin{tabular}{lccc}
\hline Factor & Odds ratio & $95 \%$ CI & $p$ value \\
\hline Duration of diabetes $>23$ years & 1.88 & $1.15-3.50$ & 0.011 \\
Uraemia & 2.43 & $1.33-4.45$ & 0.004 \\
Oedema & 2.51 & $1.79-3.54$ & 0.000 \\
Foot deformity & 1.69 & $1.08-2.63$ & 0.021 \\
Toe pressure $<30 \mathrm{mmHg}$ & 1.70 & $1.20-2.40$ & 0.003 \\
Intermittent claudication & 1.88 & $1.25-2.82$ & 0.002 \\
Rest pain & 2.06 & $1.45-2.98$ & 0.000 \\
Multiple ulcers & 2.92 & $1.90-4.49$ & 0.000 \\
Non-compliant & 2.15 & $1.26-3.66$ & 0.005 \\
Male sex & 1.51 & $1.06-2.15$ & 0.021 \\
\hline
\end{tabular}

reporting primarily healing of $77 \%(n=1,229)$, Oyibo et al. $65 \%(n=194)$, Jeffcoate et al. $66 \%(n=449)$, Gul et al. $72 \%$ $(n=200)$ and Beckert et al. $74 \%(n=1,000)[4,18-21]$. Comparisons between studies are difficult due to differences in design, setting, patient selection, definitions, follow-up time and other confounding factors. In the present study, healing was defined as intact skin for 6 months or intact skin at time of death, whereas other studies provided no definition or failed to report follow-up. Our patients, on the other hand, were followed until healing was achieved compared with studies with an observation time of 6 to 12 months [20-22]. The trend in all these studies over time is a successive improvement in healing rate [1].

It is also important how mortality and healing are reported, but in some cohort studies patients who deceased unhealed were excluded [21]. In our study deceased patients were not excluded from evaluation and the rate of mortality in patients with unhealed ulcers $(17 \%)$ was in agreement with previous observations [3, 4]. We did not investigate the cause of death, but in a previous study from the same area, three of four deaths were of cardio- or cerebrovascular origin [23]. The deceased patients had signs of severe co-morbidity and often lived in assisted living or depended on home nursing services. The present findings indicate that the foot ulcer should be considered a sign of multi-organ disease.

\section{General factors}

Information regarding general characteristics and comorbidity from studies related to outcome of foot ulcer is varied [2]. Demographic data in the present study seem to be consistent with the Eurodiale Study with regard to the proportion of PVD and co-morbidity related to major amputation [24]. However, the inclusion of more patients with neuroischaemic/ischaemic ulcers may be explained by not excluding patients with short expected survival times [20]. In the present study, factors like age, sex and duration of diabetes did not have such an evident influence on the outcome compared with other studies [21, 22, 25-27]. Age is known to be an important factor related to progress of PVD, neuropathy and lower leg amputation $[3,28,29]$ as well as to probability of healing, [9], but this was not the case in all studies [21] and especially not in short-term observation studies of neuropathic foot ulcer $<20$ weeks $[22,26]$. In the present study $51 \%$ of patients older than 80 years of age healed without any amputation.

\section{Local factors}

It should be recognised that factors like neuropathy, deformity and trauma constitute factors related to development of ulcer [2]. In the present study most patients had 
neuropathy $(92 \%)$ and foot deformities (77\%), but this was not related to outcome. However, the protocol did not include a grading system for degree of neuropathy or extent of foot deformity. Duration of ulcer before presenting at the foot clinic was not related to its outcome. Duration of ulcer has been related to outcome in cohorts/trials of neuropathic diabetic foot ulcers in an observation of $<20$ weeks [22]. A correlation between duration of ulcer and healing time has been established, but the question of whether duration is related to outcome in unselected diabetic foot ulcer is more controversial. Cause and site of ulcer did not have evident influence on outcome compared with extent of tissue involved when analysing the whole cohort, but outcome was strongly related to severity of ulcer. Ulcer area as a determinant factor on outcome was not identified in our study, although this has been suggested by others [27]. However, these data must be interpreted with caution due to methodological issues regarding ulcer size and volume. In the present study oedema was related to outcome both in neuropathic and neuroischaemic ulcers. Oedema is usually of multi-factorial origin and should be treated accordingly $[2,30]$.

\section{Neuropathic ulcers}

In the present study, deep foot infection, site of ulcer (plantar for foot ulcer, metatarsal head) and co-morbidity (non-retinal eye disease, end-stage renal disease, oedema, walking disability) were related to minor or major amputation in non-ischaemic patients. Diabetes with neuropathy and a long-standing plantar ulcer increases the risk of infection and was an independent risk factor for foot infection in a prospective study [31]. An infected foot ulcer precedes about two thirds of amputations according to benchmarking studies [32] and infection was the immediate cause for amputation with or without ischaemia in more than $50 \%$ of diabetes-related amputations [16,33]. The Eurodiale Study showed $58 \%$ prevalence of infection in all ulcer patients admitted to foot centres in Europe [24]. Although foot infections are severe with potentially disastrous consequences, both for limb and individual, choice of treatment is still empirical and most studies of antibiotics in diabetic foot infections include skin infection only and not ulcers with deep infections [2]. The question of which factors determine whether patients are infected is controversial, and studies with hard endpoints on outcome of infections in diabetic foot ulcers are limited in number $[2,34,35]$. The total number of infections in our study may be underestimated, as our protocol only included deep infections.

Neuroischaemic/ischaemic ulcer

In the present study, $36 \%$ of neuroischaemic/ischaemic ulcers healed primarily, while multiple ulcer, uraemia, extent of PVD and tissue loss were the most important factors for the probability of major amputation. Our findings regarding the proportion of neuroischaemic/ ischaemic ulcers emphasises those of the Eurodiale Study, in which $49 \%$ of diabetes patients with foot ulcer admitted to a foot clinic had signs of PVD [24]. We did not exclude patients with expected short survival, but still in the present study $49 \%$ of surviving patients had signs of severe PVD. PVD, when present, is strongly related to risk of major amputation and constitutes the most important factor related to outcome in neuroischaemic/ischaemic foot ulcers $[1,8$, 28]. Despite the fact that symptoms like claudication and rest pain are often not present, in most studies PVD was not analysed with non-invasive vascular testing (transcutaneous oxygen and carbon pressure, toe pressure, ankle pressure) [2]. Rest pain as defined according to protocol did not consider cause of pain, and this factor needs to be explored further. PVD has also been associated with a twofold increase of foot infections [31] and 31\% of patients in the Eurodiale Study had both PVD and infection [24]. The interaction between ischaemia and infection in the neuroischaemic foot [36] needs to be explored further.

In the neuroischaemic/ischaemic group male sex was related to increased probability of amputation. This is in agreement with some $[9,18,37]$ but not all $[21,36]$ studies. PVD and lower leg amputation have been considered more common among men than women, as well as presence of plantar forefoot ulcer and infection [5, 28, 38-40], but not consistently so [4]. If there is a real difference between men and women regarding wound healing and probability of amputation in the diabetic foot, there are various possible reasons: genetic (different candidate genes increase the risk of late complications in diabetes) [41]; hormonal factors (with oestrogen protecting women from vascular comorbidity and making for potential differences in immune competence) [42]; behavioural differences (smoking habits, risk-taking life style, attitudes to health and care-seeking behaviour) [43]; and differences in life expectancy.

Non-compliance in a patient with diabetes and foot ulcer as a risk factor for amputation is difficult to distinguish from true neglect and lack of awareness due to complications such as neuropathy and visual impairment [2]. Potential communication bias between healthcare providers and patients highlights the need for individually adjusted education and improved communication methods for nurses providing life style-changing patient education [36]. This provides an area of future research.

\section{Methodology}

Substantial methodological considerations need to born in mind when evaluating the present cohort study. Potential negative selection bias always has to be considered, since 
the patients were admitted to a university-based foot centre and no exceptions were made with regard to age, comorbidity or expected survival. It cannot be excluded that more superficial ulcers of neuropathic origin were treated in primary healthcare without the knowledge of the foot team. There was a general agreement at the hospital, that irrespective of to whom patients were admitted, they would be passed on to the foot centre. Definition of healing varies between studies and we considered 6 months necessary to prove patients remained healed. Definitions of outcome can be very complex, an issue that has been previously analysed [4]. Patients in our study were divided into groups according to outcome: primary healed, minor amputation, major amputation and patients deceased unhealed. In some studies minor amputations are included in primary healed and also the definition of amputation varies. Comparisons between centres are difficult, due to the heterogeneity of patients and complexity of the disease [44]. In many healthcare systems there are limited possibilities for following up patients until healing is achieved. The Swedish system, due to its geographical responsibilities and reimbursement system, enables patients to be followed up until a specific endpoint, irrespective of who the careprovider is [45]. This may explain the present drop-out rate of $1.2 \%$. Our patients seem comparable to the Eurodiale Study with regard to demographic data and healing frequency in spite of their short follow-up time (12 months) [24].

A large amount of missing data on retinopathy was mainly due to patients being unable to attend fundoscopy. Duration of diabetes with 0 years of duration is misleading. What is meant is that patients were diagnosed with diabetes when presenting with a foot ulcer and the true duration is unknown. Duration of ulcer when presenting at the foot centre is the most uncertain variable, due to a combination of patients' unawareness of the need to seek healthcare and healthcare providers' choice of treatment and willingness to refer patients [46]. Twenty per cent of the major amputated patients and $38 \%$ of the deceased patients lived in assisted living before ulceration, with healthcare professionals involved in their daily life. It is uncertain how the ulcer was treated before referral to the foot centre, due to lack of proper documentation from the home nursing services. We did not include deceased patients in the regression analysis, as we cannot estimate whether they would have healed with or without minor or major amputation, if they had survived.

\section{Conclusion}

Patients with diabetes and foot ulcer are suffering from multi-organ disease. There is a complexity of factors related to outcome, of which co-morbidity, infection and ischaemia are the most important. Probability of amputation is increased in neuropathic ulcers by deep foot infection, location and co-morbidity. Patients with neuroischaemic ulcers are more common than previously described, and comorbidity, extent of PVD and type of ulcer are the most important factors for the probability of healing with amputation. In future research on prevention and management of diabetic foot ulcers it will be important to distinguish between neuropathic and neuroischaemic/ ischaemic ulcers.

Acknowledgements This study was supported by grants from the Research Funds Malmö University Hospital and the Skåne Research Foundation. M.-B. Johansson, I. Dupros, G. Larsson, Å. Asmundsson, L. K. Bengtsson and M. Jonsson helped us by providing data for the database. S. Gershater provided data management support.

Duality of interest The authors declare that there is no duality of interest associated with this manuscript.

\section{References}

1. Boulton AJM, Vileikyte L, Ragnarson-Tennvall G, Apelqvist J (2005) The global burden of diabetic foot disease. Lancet $366: 1719-1724$

2. International Working Group on the Diabetic Foot/Consultative Section of the IDF (2007) Practical guidelines in the management and the prevention of the diabetic foot, based upon the International Consensus on the Diabetic Foot (DVD)

3. Larsson J, Agardh CD, Apelqvist J, Stenström A (1998) Longterm prognosis after healed amputation in patients with diabetes. Clin Orthop Relat Res 350:149-158

4. Jeffcoate WJ, Chipchase SY, Ince P, Game FL (2006) Assessing the outcome of the management of diabetic foot ulcers using ulcer-related and person-related measures. Diabetes Care 29:1784-1787

5. Singh N, Armstrong DG, Lipsky BA (2005) Preventing foot ulcers in patients with diabetes. JAMA 293:217-228

6. Apelqvist J (2007) Diabetic foot ulcers: Evidence, cost and management. Diabet Foot J 10:6-8

7. Wagner FW (1981) The dysvascular foot: a system for diagnosis and treatment. Foot Ankle 2:64-122

8. Apelqvist J, Castenfors J, Larsson J, Stenström A, Agardh C (1989) Wound classification is more important than site of ulceration in the outcome of diabetic foot ulcers. Diabet Med 6:526-530

9. Apelqvist J, Agardh CD (1992) The association between clinical risk factors and outcome of diabetic foot ulcers. Diabetes Res Clin Pract 18:43-53

10. Colwell JA, Bingham SF, Abraira C, Anderson JW, Kwaan HC (1985) V.A. Cooperative Study on antiplatelet agents in diabetic patients after amputation for gangrene: III. Definitions and review of design and baseline characteristics. Horm Metab Res Suppl 15:69-73

11. Boulton A, Connor H, Cavanagh PR (eds) (2000) The foot in diabetes. Wiley, Chichester

12. Cannon J (1965) Intermittent claudication what it is and isn't. Calif Med 102:301-305

13. Apelqvist J, Castenfors J, Stenström A, Agardh C (1989) Prognostic value of systolic ankle and toe pressure levels in outcome of diabetic foot ulcer. Diabetes Care 12:373-378 
14. Eneroth M, Larsson J, Oscarsson C, Apelqvist J (2004) Nutritional supplementation for diabetic foot ulcers: the first RCT. J Wound Care 13:230-234

15. Eneroth M, Larsson J, Apelqvist J (1999/0) Deep foot infections in patients with diabetes and foot ulcer: an entity with different characteristics, treatments, and prognosis. J Diabetes Its Complicat 13:254-263

16. Larsson J, Agardh C, Apelqvist J, Stenström A (1995) Clinical characteristics in relation to final amputation level in diabetic patients with foot ulcers: a prospective study of healing below or above the ankle in 187 patients. Foot Ankle 16:69-74

17. Moore DS, McCabe GP (2006) Introduction to the practice of statistics. Freeman, New York

18. Oyibo SO, Jude EB, Tarawneh I et al (2001) The effects of ulcer size and site, patient's age, sex and type and duration of diabetes on the outcome of diabetic foot ulcers. Diabet Med 18:133-138

19. Gul A, Basit A, Ali SM, Ahmadani MY, Miyan Z (2006) Role of wound classicifation in predicting the outcome of diabetic foot ulcer. J Pak Med Assoc 56:444-447

20. Prompers L, Schaper N, Apelqvist J et al (2008) Prediction of outcome in individuals with diabetic foot ulcers: focus on the differences between individuals with and without peripheral arterial disease. The Eurodiale study. Diabetologia 51:747-755

21. Beckert S, Witte M, Wicke C, Konigsrainer A, Coerper S (2006) A new wound-based severity score for diabetic foot ulcers: a prospective analysis of 1,000 patients. Diabetes Care 29:988-992

22. Margolis DJ, Allen-Taylor L, Hoffstad O, Berlin JA (2002) Diabetic neuropathic foot ulcers: the association of wound size, wound duration, and wound grade on healing. Diabetes Care 25:1835-1839

23. Apelqvist J, Larsson J, Agardh C (1993) Long-term prognosis for diabetic patients with foot ulcers. J Intern Med 233:485-491

24. Prompers L, Huijberts M, Apelqvist J et al (2007) High prevalence of ischaemia, infection and serious comorbidity in patients with diabetic foot disease in Europe. Baseline results from the Eurodiale Study. Diabetologia 50:18-25

25. Boyko EJ, Ahroni JH, Cohen V, Nelson KM, Heagerty PJ (2006) Prediction of diabetic foot ulcer occurrence using commonly available clinical information: the Seattle diabetic foot study. Diabetes Care 29:1202-1207

26. Margolis DJ, Kantor J, Santanna J, Strom BL, Berlin JA (2000) Risk factors for delayed healing of neuropathic diabetic foot ulcers: a pooled analysis. Arch Dermatol 136:1531-1535

27. Sheehan P, Jones P, Caselli A, Giurini JM, Veves A (2003) Percent change in wound area of diabetic foot ulcers over a 4week period is a robust predictor of complete healing in a 12week prospective trial. Diabetes Care 26:1879-1882

28. Bild DE, Selby JV, Sinnock P, Browner WS, Braveman P, Showstack JA (1989) Lower-extremity amputation in people with diabetes. Epidemiology and prevention. Diabetes Care 12:24-31

29. Kreines K, Johnson E, Albrink M et al (1985) The course of peripheral vascular disease in non-insulin-dependent diabetes. Diabetes Care 8:235-243
30. Edmonds M (1986) The diabetic foot: pathophysiology and treatment. Clin Endocrinol Metab 15:889-916

31. Lavery LA, Armstrong DG, Wunderlich RP, Mohler MJ, Wendel CS, Lipsky BA (2006) Risk factors for foot infections in individuals with diabetes. Diabetes Care 29:1288-1293

32. Pecoraro RE, Reiber GE, Burgess EM (1990) Pathways to diabetic limb amputation. Basis for prevention. Diabetes Care 13:513-521

33. Knighton DR, Fylling CP, Fiegel VD, Cerra F (1990) Amputation prevention in an independently reviewed at-risk diabetic population using a comprehensive wound care protocol. Am J Surg 160:466-471

34. Lazzarini L, Lipsky BA, Mader JT (2005) Antibiotic treatment of osteomyelitis: what have we learned from 30 years of clinical trials? Int J Infect Dis 9:127-138

35. Apelqvist J (2008) The foot in perspective. Diabetes/Metab Res Rev 24(Suppl 1):S110-S115

36. Edmonds M, Boulton A, Buckenham T et al (1996) Report of the diabetic foot and amputation group. Diabet Med 13:S27S42

37. Marston WA (2006) Risk factors associated with healing chronic diabetic foot ulcers: the importance of hyperglycaemia. Ostomy Wound Manage 52:26-28

38. Beach KW, Strandness DEJ (1980) Arteriosclerosis obliterans and associated risk factors in insulin-dependent and non-insulindependent diabetes. Diabetes 29:882-888

39. Kuebler TW, Bendick PJ, Fineberg SE et al (1983) Diabetes mellitus and cerebrovascular disease: prevalence of carotid artery occlusive disease and associated risk factors in 482 adult diabetic patients. Diabetes Care 6:274-278

40. Nelson RG, Gohdes DM, Everhart JE et al (1988) Lowerextremity amputations in NIDDM. 12-yr follow-up study in Pima Indians. Diabetes Care 11:8-16

41. Lindholm E, Melander O, Almgren P et al (2006) Polymorphism in the MHC2TA gene is associated with features of the metabolic syndrome and cardiovascular mortality. PLoS ONE 1 (e64):1-5

42. Tivesten $\AA$, Mellström D, Jutberger H et al (2007) Low serum testosterone and high serum estradiol associate with lower extremity peripheral arterial disease in elderly men: the MrOS Study in Sweden. J Am Coll Cardiol 50:1070-1076

43. Hjelm K, Nyberg P, Apelqvist J (2002) Gender influences beliefs about health and illness in diabetic subjects with severe foot lesions. J Adv Nurs 40:673-684

44. Connor H (1999) Diabetic foot disease - where is the evidence? Diabet Med 16:799-800

45. Norlund A, Apelqvist J, Bitzén P, Nyberg P, Scherstén B (2001) Cost of illness of adult diabetes mellitus underestimated if comorbidity is not considered. J Intern Med 250:57-65

46. Apelqvist J, Larsson $J$ (2000) What is the most effective way to reduce incidence of amputation in the diabetic foot? Diabetes Metab Res Rev 16:S75-S83 